\title{
Errare humanum est, sed in errare perseverare diabolicum: methodological errors in the assessment of the relationship between I-131 therapy and possible increases in the incidence of malignancies
}

\author{
Frederik A. Verburg ${ }^{1,2}$. Martha Hoffmann ${ }^{1,3}$ • Ioannis lakovou ${ }^{1,4}$ • Mark W. Konijnenberg ${ }^{5,6}$ Jasna Mihailovic ${ }^{1,7,8}$. \\ Pablo Minguez Gabina ${ }^{5,9}$ - Petra Petranović Ovčariček ${ }^{1,10}$ - Cristoph Reiners $^{11}$ • Alexis Vrachimis ${ }^{1,12}$. \\ Slimane Zerdoud ${ }^{1,13} \cdot$ Luca Giovanella $^{1,14,15}$ - Markus Luster ${ }^{1,2}$
}

Published online: 5 December 2019

(C) Springer-Verlag GmbH Germany, part of Springer Nature 2019

For nearly eight decades [1] now, radioactive iodine (RAI) therapy has been playing an important role in the treatment of benign thyroid diseases (BTD) as well as differentiated thyroid cancer (DTC) [2].

Originally hailed in the popular press as a form of magic, it quite soon emerged that even this very specific, targeted drug is associated with some long-term side effects and complications. The first reports of acute myeloid leukemia in DTC patients treated with high activity RAI were published in the 1950s [3]. Certainly, the hypothesis that exposure to RAI, like with any form of radiation, might cause an increase in the rate of secondary hematological malignancies has a certain logic to it, as I-131 will, after administration, first circulate systemically before being taken up by cells of thyroid follicular lineage.

This article is part of the Topical Collection on Editorial

Frederik A. Verburg

verburg@med.uni-marburg.de

EANM Thyroid Committee, Vienna, Austria

2 Department of Nuclear Medicine, University Hospital Marburg, Baldinger Straße, 35043 Marburg, Germany

3 Department of Nuclear Medicine, Radiology Center, Vienna, Austria

4 Academic Department of Nuclear Medicine, Aristotle University, Thessaloniki, Greece

5 EANM Dosimetry Committee, Vienna, Austria

6 Department of Radiology \& Nuclear Medicine, Erasmus Medical Center, Rotterdam, The Netherlands

7 Department of Radiology, Faculty of Medicine, University of Novi Sad, Novi Sad, Serbia

8 Department of Nuclear Medicine, Oncology Institute of Vojvodina, Sremska Kamenica, Serbia
Both non-target organs expressing the sodium-iodide symporter such as the stomach lining, the salivary glands, or mammary gland tissue and well-perfused organs such as the bone marrow are therefore exposed to significant radiation doses when a patient is exposed to I-131 - as was, e.g., already shown in the 1960s for the bone marrow [4]. However, although only based on a few patients, there is also some evidence that the DNA damage to, e.g., the blood cells can be repaired effectively after RAI [5].

Over the course of decades, I-131 has time and again been the subject of scrutiny regarding its potential for the induction of secondary malignancies. However, few, if any, studies have rigorously observed all the criteria needed to prove a relationship between I-131 and cancer incidence. To establish whether

9 Department of Medical Physics and Radiation Protection, Gurutzeta/ Cruces University Hospital, Barakaldo, Spain

10 Department of Oncology and Nuclear medicine, University Hospital Center "Sestre milosrdnice", Zagreb, Croatia

11 Department of Nuclear Medicine, Würzburg University Hospital, Würzburg, Germany

12 Department of Nuclear Medicine, German Oncology Center, University Hospital of the European University, Limassol, Cyprus

13 Department of Nuclear Medicine, Nuclear Oncology and Thyroidological Oncology, University Cancer Institute - Oncopole, Toulouse, France

14 Clinic for Nuclear Medicine and Competence Centre for Thyroid Diseases, Centre Imaging Institute of Southern Switzerland, Bellinzona, Switzerland

15 Clinic for Nuclear Medicine, Zurich University Hospital, Zurich, Switzerland 
there is a relationship between radiation exposure and the risk of malignancy due to this exposure, the following difficulties and requirements should be taken into account:

1. The age at the time of radiation exposure is of great importance, as the risk of radiation induced malignancies is considerably higher in children/adolescents than in adults.

2. Since it is well known that radiation-induced malignancies develop after a latency time of approximately $2-3$ years for hematologic malignancies and approximately 5 years for solid cancers, this "lag time" has to be taken into consideration. For most of those malignancies, the risk remains elevated lifelong.

3. A dose dependency should be established, as a higher absorbed radiation dose by the organs at risk should be associated with a higher risk of malignant diseases.

4. A control group consisting of completely healthy subjects is insufficient for proving an increased incidence or mortality due to malignant diseases in patients treated with radiation for a given disease. To conclusively prove such an effect, the study population should be compared with an age- and sex-matched control group consisting of patients with the same thyroid disease, who were treated by non-radioactive procedures (e.g., surgery) or not treated at all.

Unfortunately, as illustrated in a previous review [6], thus far, no studies proving a relationship between I-131 therapy and an elevated malignancy risk fulfilling all these methodological criteria are available for I-131 therapy of benign thyroid disease. Furthermore, the only malignancy where such an elevated risk was generally considered proven for the much higher activities associated with DTC therapy concerned acute myeloid leukemia.

Recently, however, two studies have come out again implicating I-131 therapy for malignant and benign thyroid disease as a cause for additional cases of malignancy [7-9]. Not only were these studies controversial because of the message- - I131 therapy causes cancer - both of these tried to convey but also because of their methodological flaws.

The first study, published in two different versions with essentially the same message by Molenaar et al. [7, 8], alleged that I-131 therapy of DTC was associated with if not causative of an elevated risk of hematological malignancies. Much has been stated about the methodological flaws of this study in a host of letters to the editors of both respected journals and we have commented on this paper before in the present journal [10-12]. Suffice it to say that save for a more or less adequate choice of control group, this paper has no redeeming methodological qualities in terms of the first three criteria listed above.

A few weeks ago, a paper exploring the relationship between I-131 therapy of benign thyroid disease and the incidence of malignancies after this therapy was published [9]. This paper, which has a host of methodological deficits, was co-authored by some of the most vocal critics of the papers by
Molenaar et al. [13]. Hence, it is all the more surprising that, even with a benevolent mindset, this paper cannot be described as methodologically accurate and factual in its conclusions.

Of course, we will not deny that the authors have undertaken a fiendishly difficult task in reconstructing and following a patient cohort initially treated between 1946 and 1964. Certainly, the study, which is an extension of one of the better papers published thus far on the subject of a non-causal relationship between I-131 therapy for benign disease and secondary cancers, [14] is not without its attempts to at least get some aspects of the methodology correct. The authors do consider the patients' individual age and year of birth as well as other demographic factors. The authors do account for a latency time of 5 years. And they certainly make an attempt to examine a dose-response relationship by using a dosimetric model from which they derive standard average values for the radiation exposure of various organs based on the administered activity. Using this model, they provide a risk estimation of future cancer deaths (contrary to observed events in their previous study by Ron et al. [14]) attributable to radiation exposure on the basis of a hypothetical patient population and they conclude that there is a dose-response relationship between the occurrence of death due to all solid cancers in aggregate and the radiation absorbed whole body dose as well as between death due to breast cancer and the radiation absorbed dose. However, as a surrogate for the whole body absorbed dose, the authors used the stomach dose. To the best of our knowledge, the use of the stomach absorbed dose as such is not a common practice. And even if this might be justifiable for other sources of radiation, for I-131, it most probably is not. Obviously, the assumption that the stomach dose can be used as a surrogate for the whole body dose is not based on imaging, but on an evaluation of the extracellular fluid distribution. Should this be true, the post therapy I-131 image would show more than thyroid (remnant), stomach, and bowel uptake. However, unlike most other tissues in the body, the stomach lining actually shows a strong expression of the sodium iodide symporter, actively taking up I-131. The dosimetry data for medium thyroid uptake by I-131 iodide according to ICRP-128 consequently shows a factor 5-6 lower doses to the breast. Therefore, the stomach dose is probably representative of the whole body dose, although it remains difficult to deduce how the use of a more realistic substitute such as the liver or the blood dose might affect the results.

Furthermore, the authors unfortunately have made no comparison with a group of comparable patients not treated with I131 (e.g., with surgery and/or anti-thyroid drugs). Therefore, the title of the article "association of RAI treatment with cancer mortality in patients with hyperthyroidism" is not just misleading, but also plainly and factually wrong. The only result the authors show is that there is a positive correlation or association between an increasing estimated radiation dose 
absorbed by the whole body or specific organs and the rate of cancer deaths. However, without a proper control group, it remains pure speculation whether this effect is causative, or just indicative.

For years, it has been established that both Graves' disease [15] and hyperthyroidism in general [16] are causative of an increasing cancer incidence. As larger thyroids and more severe hyperthyroidism in Graves' disease are in clinical practice associated with higher activities necessary for effective treatment, it could very well be that it is the hyperthyroidism per se that is causing the excess cancer mortality - there is a considerable body of evidence showing this. A recent study from the epidemiological Rotterdam study showed even that elevated thyroid hormone level itself was associated with an increase in breast cancer-related mortality [17] —at nearly precisely the same level as Kitahara et al. [9] purport to show for RAI. When one, however, regards the increasing I-131 activity as a proxy for data which during the recruitment period could not yet be determined with the accuracy required and possible today, such as a thyroid volume determined by imaging or levels of thyroid-related hormones in the blood and most importantly - quantitative measurement of thyroid uptake and effective half-life, the real cause for the increased cancer mortality could very well be the hyperthyroidism itself.

It is also remarkable that the group of patients receiving the highest tertile of I-131 activities also had a much higher rate of coronary, hypertensive, and other types of heart disease. All these diseases are associated with smoking (which might not have been recorded yet in the recruitment period of the study as the detrimental effects of smoking were not yet widely known in those days). The study cohort nearly exclusively consisted of patients with Graves' disease. It is well-known that smoking is associated with a more severe course of Graves' disease. Therefore, it is quite conceivable that again, the I-131 activity is merely a proxy for other underlying effects. For instance, heavier smokers will probably suffer from more severe Graves' disease and will therefore both receive more I-131, and will be at higher cancer risk due to the wellknown oncogenic effects of tobacco consumption. Furthermore, besides smoking, the results were also not adjusted for obesity, alcohol use, or reproductive factors and, as stated by the authors in the discussion section, confounding by these known cancer risk factors is possible.

Also, it has to be noted that anti-thyroid (ATD) drugs may play a considerable role in disease-related mortality. In fact, a hint in this direction was again given by two of the co-authors of the paper under discussion here, who published additional data and analyses [18] which were, either unconsciously or consciously, withheld from the original paper by Kitahara et al. Here, Tulchinsky and Brill show that the SMR for breast cancer is higher for patients treated with ATD alone than in patients treated with RAI therapy.
Certainly, the study by Kitahara et al. [9] is again a fine example of a methodologically insufficient study nonetheless drawing wide-ranging conclusions which could and will, if not seen in the proper light, lead to potentially detrimental changes in therapy regimens of patients with hyperthyroidism. It remains therefore the case that in spite of nearly eight decades of intensive clinical use, still no study exists in literature which both shows a positive, oncogenic effect of I-131 therapy of benign thyroid disease and passes rigorous scrutiny of methodological sufficiency.

Hence, until evidence from randomized trials has assessed the long-term prognostic impact of RAI, we are convinced and still believe that, as stated before for cancer [10, 19], postoperative RAI remains an eminently sensible idea, not just for most DTC patients but also for patients with benign thyroid disease.

\section{Compliance with ethical standards}

Conflict of interest The authors declare that they have no conflict of interest.

\section{References}

1. Hertz S, Roberts A. Application of radioactive iodine in Graves disease. J Clin Invest. 1942;21:624.

2. Seidlin SM, Marinelli LD, Oshry E. Radioactive iodine therapy: effect on functioning metastases of adenocarcinoma of the thyroid. JAMA. 1946;132:838-47.

3. Seidlin SM, Siegal E, Yalow AA, Melamed S. Acute myeloid leukemia following prolonged iodine-131 therapy for metastatic thyroid carcinoma. Science. 1956;123:800-1.

4. Benua RS, Cicale NR, Sonenberg M, Rawson RW. The relation of radioiodine dosimetry to results and complications in the treatment of metastatic thyroid cancer. Am J Roentgenol Radium Therapy, Nucl Med. 1962;87:171-82.

5. Eberlein U, Scherthan H, Bluemel C, Peper M, Lapa C, Buck AK, et al. DNA Damage in peripheral blood lymphocytes of thyroid cancer patients after radioiodine therapy. J Nucl Med. 2016;57: 173-9.

6. Verburg FA, Luster M, Lassmann M, Reiners C. (131) I therapy in patients with benign thyroid disease does not conclusively lead to a higher risk of subsequent malignancies. Nuklearmedizin. 2011;50: 93-9.

7. Molenaar RJ, Sidana S, Radivoyevitch T, Advani AS, Gerds AT, Carraway HE, et al. Risk of hematologic malignancies after radioiodine treatment of well-differentiated thyroid cancer. J Clin Oncol. 2018;36:1831-9.

8. Molenaar RJ, Pleyer C, Radivoyevitch T, Sidana S, Godley A, Advani AS, et al. Risk of developing chronic myeloid neoplasms in well-differentiated thyroid cancer patients treated with radioactive iodine. Leukemia. 2018;32:952-9.

9. Kitahara CM, Berrington de Gonzalez A, Bouville A, Brill AB, Doody MM, Melo DR, et al. Association of radioactive iodine treatment with cancer mortality in patients with hyperthyroidism. JAMA Intern Med. 2019;179:1034.

10. Verburg FA, Giovanella L, Iakovou I, Konijnenberg MW, Langsteger W, Lassmann M, et al. I-131 as adjuvant treatment for differentiated thyroid carcinoma may cause an increase in the 
incidence of secondary haematological malignancies: an "inconvenient" truth? Eur J Nucl Med Mol Imaging. 2018;45:2247-9.

11. Piccardo A, Puntoni M, Verburg FA, Luster M, Giovanella L. Power of absolute values to avoid data misinterpretations: the case of radioiodine-induced leukemia and myelodysplasia. J Clin Oncol. 2018;36:1880-1.

12. Hindié E, Récher C, Zerdoud S, Leenhardt L, Avram AM. Risk of hematologic malignancies after radioactive iodine treatment of thyroid cancer: an unjustified warning. J Clin Oncol. 2018;36:1881-2.

13. Tulchinsky M, Baum RP, Bennet KG, Freeman LM, Jong I, Kairemo K, et al. Well-founded recommendations for radioactive iodine treatment of differentiated thyroid cancer require balanced study of benefits and harms. J Clin Oncol. 2018;36:1887-8.

14. Ron E, Doody MM, Becker DV, Brill AB, Curtis RE, Goldman $\mathrm{MB}$, et al. Cancer mortality following treatment for adult hyperthyroidism. JAMA. 1998;280:347-55.

15. Chen Y-K, Lin C-L, Chang Y-J, Cheng FT-F, Peng C-L, Sung F-C, et al. Cancer risk in patients with Graves' disease: a nationwide cohort study. Thyroid. 2013;23:879-84.
16. Krashin E, Piekiełko-Witkowska A, Ellis M, Ashur-Fabian O. Thyroid hormones and cancer: a comprehensive review of preclinical and clinical studies. Front Endocrinol (Lausanne). 2019;10:59.

17. Khan SR, Chaker L, Ruiter R, Aerts JGJV, Hofman A, Dehghan A, et al. Thyroid function and cancer risk: the Rotterdam study. J Clin Endocrinol Metab. 2016;101:5030-6.

18. Tulchinsky M, Brill AB. Spotlight on the association of radioactive iodine treatment with cancer mortality in patients with hyperthyroidism is keeping the highest risk from antithyroid drugs in the blind spot. Clin Nucl Med. 2019;44:789-91.

19. Verburg FA, Dietlein M, Lassmann M, Luster M, Reiners C. Why radioiodine remnant ablation is right for most patients with differentiated thyroid carcinoma. Eur J Nucl Med Mol Imaging. 2009;36: $343-6$.

Publisher's note Springer Nature remains neutral with regard to jurisdictional claims in published maps and institutional affiliations. 